Provided for non-commercial research and education use. Not for reproduction, distribution or commercial use.

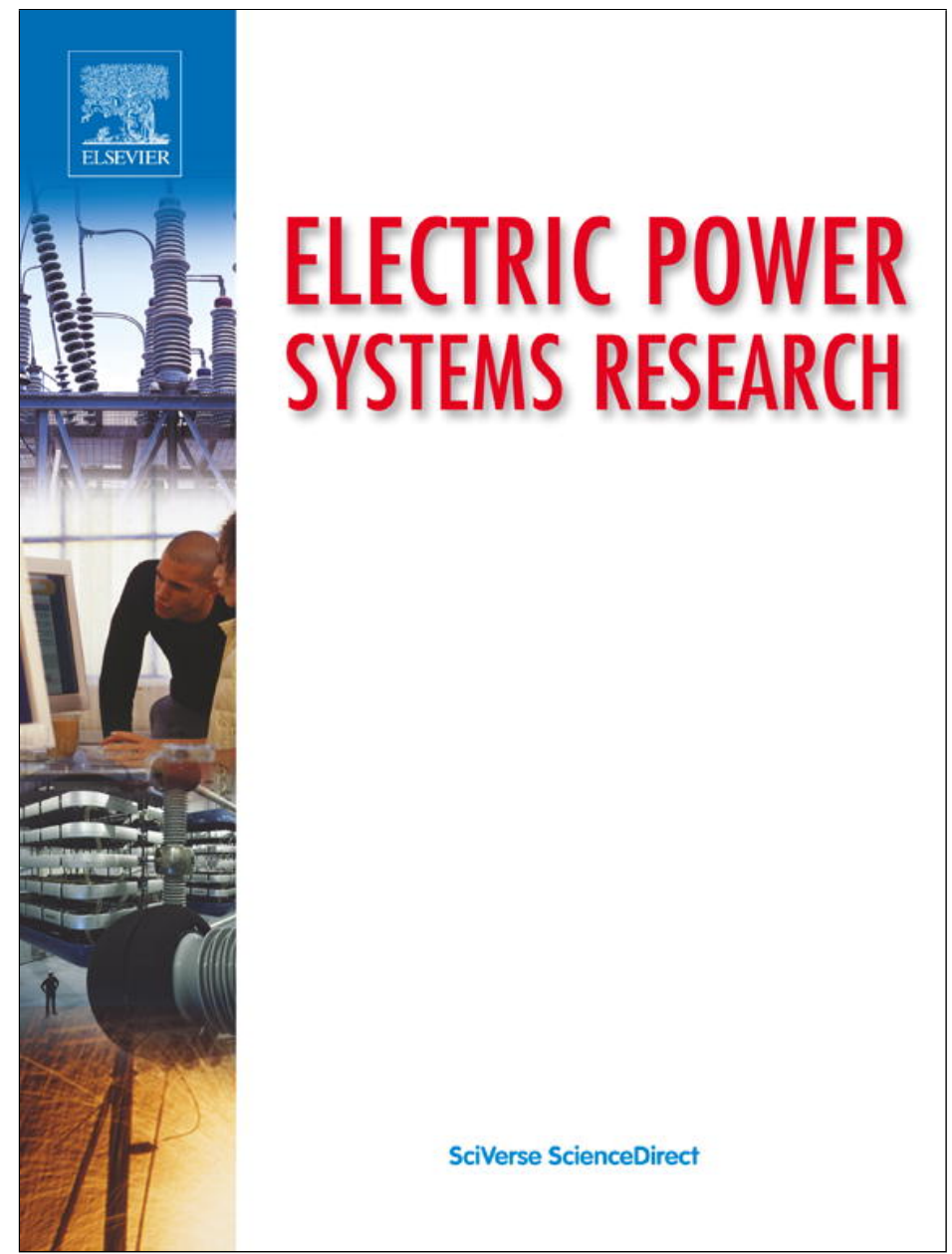

(This is a sample cover image for this issue. The actual cover is not yet available at this time.)

This article appeared in a journal published by Elsevier. The attached copy is furnished to the author for internal non-commercial research and education use, including for instruction at the authors institution and sharing with colleagues.

Other uses, including reproduction and distribution, or selling or licensing copies, or posting to personal, institutional or third party websites are prohibited.

In most cases authors are permitted to post their version of the article (e.g. in Word or Tex form) to their personal website or institutional repository. Authors requiring further information regarding Elsevier's archiving and manuscript policies are encouraged to visit:

http://www.elsevier.com/copyright 


\title{
Experiments with the interior-point method for solving large scale Optimal Power Flow problems
}

\author{
Florin Capitanescu ${ }^{\mathrm{a}, *}$, Louis Wehenkel ${ }^{\mathrm{b}}$ \\ ${ }^{a}$ University of Luxembourg, Interdisciplinary Centre for Security, Reliability and Trust (SnT), 6, rue Richard Coudenhove-Kalergi, L-1359 Luxembourg, Luxembourg \\ ${ }^{\mathrm{b}}$ University of Liège, Institut Montefiore, Sart-Tilman B28, B-4000 Liège, Belgium
}

\section{A R T I C L E I N F O}

\section{Article history:}

Received 16 March 2012

Received in revised form

17 September 2012

Accepted 1 October 2012

\begin{abstract}
A B S T R A C T
This paper reports extensive results obtained with the interior-point method (IPM) for nonlinear programmes (NLPs) stemming from large-scale and severely constrained classical Optimal Power Flow (OPF) and Security-Constrained Optimal Power Flow (SCOPF) problems. The paper discusses transparently the problems encountered such as convergence reliability and speed issues of the method.
\end{abstract}

(C) 2012 Elsevier B.V. All rights reserved.

\section{Keywords:}

Optimal Power Flow

Security-Constrained Optimal Power Flow

Interior-point method

Nonlinear programming

\section{Introduction}

The Optimal Power Flow (OPF) [1] is a static, nonlinear, nonconvex, large-scale optimization problem with continuous and discrete variables. The Security-Constrained Optimal Power Flow (SCOPF) is a generalization of the OPF problem that ensures additionally the system security with respect to a set of postulated contingencies [2]. OPF is nowadays an essential tool in power systems planning, operational planning and real-time operation.

Due to critical environmental issues, nowadays most power systems have to accommodate a significant level of penetration of renewable intermittent generation. This requires smarter ways of control in real-time according to the principles: just-in-time, just-in-place, and just-in-context [3]. Since a classical day-ahead preventive SCOPF approach would be not anymore sustainable, we foresee that the need for using an automatic adaptive optimal control scheme, and in particular a (SC)OPF in real-time, is more and more stringent. This raises the same old concerns regarding mostly the reliability and speed of such an approach [4,5]. Furthermore, in Europe there is a trend to check the security and propose coordinated control actions for a large interconnection, composed by independent power systems, in a broader way [6], which calls for

\footnotetext{
* Corresponding author. Tel.: +352 466644 5345; fax: +352 4666445669.

E-mail addresses: florin.capitanescu@uni.lu (F. Capitanescu), l.wehenkel@ulg.ac.be (L. Wehenkel).
}

tools able to deal with very large scale optimization problems, with up to hundreds of millions of variables and constraints.

IPM $[7,8]$ has been successfully applied for nearly two decades to various OPF problems [9-13]. The main advantages of the IPM are: (i) ease of handling inequality constraints by logarithmic barrier functions, (ii) speed of convergence, and (iii) a strictly feasible initial point is not required. The drawbacks of the IPM are: (i) the heuristic to decrease the barrier parameter, (ii) the required positivity of slack variables and their corresponding dual variables at every iteration (which may drastically shorten the Newton step length), and (iii) it does not warmstart well.

Confidentiality of large scale real-life power systems data deprives the power systems community of realistic benchmarks at least in the field of OPF and prevents researchers from reporting OPF results obtained in realistic conditions and thereby the fair assessment of existing OPF methods on various different problems. Until recently, when a large 2746-bus model of the Polish power system became freely available [14], the largest test bed for the OPF/SCOPF programs was an IEEE system of 300 buses [15].

The large-scale ${ }^{1}$ NLP OPF problems tackled by IPM reported in the literature involve power systems of 2098 buses [16,17], 2256 buses [18,19], 2423 buses [9], 2746 buses [20], 2935 buses [21], 3012 buses [22], and 3467 buses [10]. Other alternative algorithms have been also used for large-scale OPF, e.g. Newton method was

\footnotetext{
${ }^{1}$ We arbitrarily consider a power system as "large" if it contains more than 2000 buses.
} 
applied to a 2436-bus grid [23], a non-interior point complementarity method was applied to a 2098-bus system [16,24], a modified barrier Lagrangian function was applied to a 2111-bus system [25], a modified barrier approach was applied to a 2256-bus system [26], and a trust-region based augmented Lagrangian method was applied to network models of 2935 buses [21] and 3012 buses [22]. Furthermore, although several commercial NLP SCOPF packages are available from various vendors [27-29], and are routinely used by many system operators, the scientific literature reporting on experiments using SCOPF solvers on large-scale systems is quite limited except of the following works: [30] uses an SLP approach for a model of 12,965 buses, [29] relies on an interior-point solver for a 2351-bus network, [31] employs a conic programming for a 2383-bus Polish system, [32] relies on the IPM for a 2746-bus Polish system, [22] applies IPM to a 3012-bus grid, [33] uses a SLP for the 3551 buses UK system, and [34] uses a method combining IPM and conjugate gradient for a 15,000 bus European system.

Motivated by the fact that these works present generally only one example of a successful application of a given algorithm to a given OPF/SCOPF problem, the main contribution of this paper lies in the transparent report of extensive results with a noncommercial IPM-based OPF program, developed by the authors for research purposes [35], on severely constrained large-scale OPF and SCOPF problems. The challenges of these computations are related to: (i) the size of our 8387-bus system, which models a large part of Europe, is roughly three times larger than previously reported IPMbased NLP OPFs, (ii) the complexity of data (e.g. coexistence of many very long lines, stemming from some network equivalents, and very short lines), (iii) the toughness of the optimization problems considered compared to the literature (e.g. often more than thousand constraints are binding at the optimum), and (iv) the number and variety of control variables (e.g. few thousands control variables for the control of both active and reactive powers are considered together). The paper also discusses in a transparent way reliability and speed issues of the IPM.

The paper is organized as follows. Section 2 introduces the OPF problems while Section 3 briefly describes the interior-point algorithm used for solving them. Section 4 provides numerical results obtained with the IPM for several OPF and SCOPF problems. Section 5 concludes.

\section{Formulation of the Optimal Power Flow problem}

For the sake of facilitating the reader' interpretation of our results as well as to make the paper self-contained we describe in this section the formulation of two classical OPF problems.

\subsection{Notations}

Let us denote by: $n, g, c, b, l, t, o, a$, and $s$ the respective numbers of: buses $(n)$, generators $(g)$, loads $(c)$, branches $(b)$, lines $(l)$, all transformers $(t)$, transformers with controllable taps $(o)$, phase shifters $(a)$, and shunt elements ( $s$ ), respectively.

We formulate the OPF problem with complex voltages expressed in rectangular coordinates $[12,35]$ :

$\underline{V}_{i}=e_{i}+j f_{i}, \quad i=1, \ldots, n$,

where $e_{i}$ and $f_{i}$ are its real and imaginary part, respectively, the voltage magnitude being:

$V_{i}=\sqrt{e_{i}^{2}+f_{i}^{2}}$

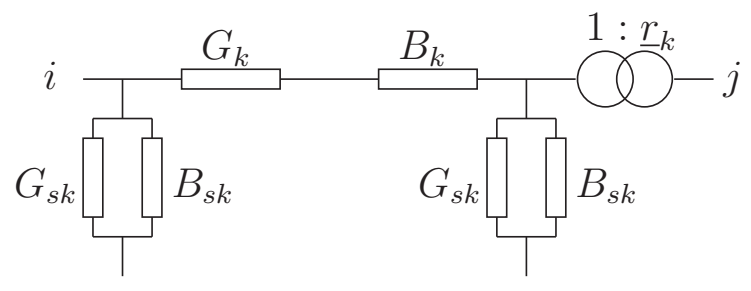

Fig. 1. Model of a generic branch.

We model any branch $k$ as a symmetrical quadruple in $\pi$ in series with an ideal transformer with complex ratio $1 / \underline{r}_{k}$ (see Fig. 1 ), where:

$\underline{r}_{k}=r_{k 1}+j r_{k 2}, \quad k=1, \ldots, b$,

$r_{k}^{2}=r_{k 1}^{2}+r_{k 2}^{2}, \quad k=1, \ldots, b$.

A transformer with tap-changer is a particular case where $r_{k 2}=0$ while a line is a particular case where $r_{k 1}=1$ and $r_{k 2}=0$.

\subsection{Objective functions}

In this paper we deal with two classical objectives namely minimum generation deviation with respect to the base case (MD):

$\mathrm{MD}=\min \sum_{i=1}^{g}\left(P_{g i}-P_{g i}^{0}\right)^{2}$,

and minimum active power losses (MPL):

$$
\begin{aligned}
\mathrm{MPL}= & \min \sum_{k=1}^{b} G_{s k} V_{i}^{2}+G_{s k} \frac{V_{j}^{2}}{r_{k}^{2}} \\
& +G_{k}\left[V_{i}^{2}+\frac{V_{j}^{2}}{r_{k}^{2}}-2 \frac{r_{k 1}\left(e_{i} e_{j}+f_{i} f_{j}\right)+r_{k 2}\left(e_{i} f_{j}-f_{i} e_{j}\right)}{r_{k}^{2}}\right],
\end{aligned}
$$

where $P_{g i}$ and $P_{g i}^{0}$ is the active power and base case active power of generator $i, G_{k}$ (respectively $G_{s k}$ ) is the conductance (respectively half shunt conductance) of the branch $k$ linking buses $i$ and $j$.

\subsection{Control variables}

We consider the following control variables: generator active power, generator reactive power, controllable transformer ratio, shunt reactance and phase shifter angle.

\subsection{Equality constraints}

Equality constraints mainly involve nodal active and reactive power balance equations, which, for the $i$ th bus $(i=1, \ldots, n)$, take on the form:

$P_{g i}-P_{c i}-\left(e_{i}^{2}+f_{i}^{2}\right) \sum_{k \in \mathcal{B}_{i}}\left(G_{s k}+G_{k}\right)$

$+\sum_{k \in \mathcal{B}_{i}}\left[\left(e_{i} e_{j_{k}}+f_{i} f_{j_{k}}\right)\left(r_{k 1} G_{k}+r_{k 2} B_{k}\right)-\left(f_{i} e_{j_{k}}-e_{i} f_{j_{k}}\right)\left(r_{k 2} G_{k}-r_{k 1} B_{k}\right)\right]=0$,

$Q_{g i}-Q_{c i}+\left(e_{i}^{2}+f_{i}^{2}\right)\left[B_{s i}+\sum_{k \in \mathcal{B}_{i}}\left(B_{s k}+B_{k}\right)\right]$

$+\sum_{k \in \mathcal{B}_{i}}\left[\left(e_{i} e_{j_{k}}+f_{i} f_{j_{k}}\right)\left(r_{k 2} G_{k}-r_{k 1} B_{k}\right)+\left(f_{i} e_{j_{k}}-e_{i} f_{j_{k}}\right)\left(r_{k 1} G_{k}+r_{k 2} B_{k}\right)=0\right.$, 
where $P_{g i}$ and $Q_{g i}$ are the active and reactive powers of the generator connected at bus $i, P_{c i}$ and $Q_{c i}$ are the active and reactive demands of the load connected at bus $i, B_{s i}$ is the shunt susceptance connected at bus $i, \mathcal{B}_{i}$ denotes the set of branches connected to bus $i$ (and $\forall k \in \mathcal{B}_{i}$, $j_{k}$ denotes the other bus to which branch $k$ is connected), $G_{k}$ and $B_{k}$ (respectively $G_{s k}$ and $B_{s k}$ ) are the longitudinal (respectively shunt) conductance and susceptance of the $k$ th branch connected to bus $i$.

Additional equality constraints may exist, as for example the setting of a phase shifter ratio to a specified reference $r_{k 0}$ :

$r_{k 1}^{2}+r_{k 2}^{2}-r_{k 0}^{2}=0, \quad k=1, \ldots, a$,

or the setting of generator voltage to a specified reference:

$e_{i}^{2}+f_{i}^{2}-\left(V_{i}^{r e f}\right)^{2}=0, \quad i=1, \ldots, g$.

\subsection{Inequality constraints}

The operational limits on (longitudinal) branches current and voltages magnitude take on the form:

$$
\begin{aligned}
& \sqrt{\left(G_{k}^{2}+B_{k}^{2}\right)\left[V_{i}^{2}+\frac{V_{j}^{2}}{r_{k}^{2}}-2 \frac{r_{k 1}\left(e_{i} e_{j}+f_{i} f_{j}\right)+r_{k 2}\left(e_{i} f_{j}-f_{i} e_{j}\right)}{r_{k}^{2}}\right]} \leq I_{k}^{\max }, \\
& k=1, \ldots, b
\end{aligned}
$$

$\left(V_{i}^{\min }\right)^{2} \leq e_{i}^{2}+f_{i}^{2} \leq\left(V_{i}^{\max }\right)^{2}, \quad i=1, \ldots, n$.

Physical limits of power system devices can be expressed as:

$P_{g i}^{\min } \leq P_{g i} \leq P_{g i}^{\max }, \quad i=1, \ldots, g$,

$Q_{g i}^{\min } \leq Q_{g i} \leq Q_{g i}^{\max }, \quad i=1, \ldots, g$,

$r_{1 i}^{\min } \leq r_{1 i} \leq r_{1 i}^{\max }, \quad i=1, \ldots, o$,

$B_{i}^{\min } \leq B_{s i} \leq B_{i}^{\max }, \quad i=1, \ldots, s$,

$\tan \phi_{i}^{\min } \leq \frac{r_{2 i}}{r_{1 i}} \leq \tan \phi_{i}^{\max }, \quad i=1, \ldots, a$,

where for the $i$ th generator $P_{g i}^{\min }, P_{g i}^{\max }$ (respectively $Q_{g i}^{\min }, Q_{g i}^{\max }$ ) are its active (respectively reactive) output limits, for the $i$ th controllable transformer $r_{1 i}^{\min }$ and $r_{1 i}^{\max }$ are bounds on its ratio, for the $i$ th shunt $B_{i}^{\min }$ and $B_{i}^{\max }$ are bounds on its susceptance, and for the $i$ th phase shifter $\phi_{i}^{\min }$ and $\phi_{i}^{\max }$ are bound on its angle.

\section{Multiple centrality corrections interior-point algorithm}

We solve the above OPF problems using the multiple centrality corrections (MCC) interior-point algorithm [17,35,36], that the authors found after extensive experiments over the years as the most reliable IPM algorithm. In order to make the paper selfcontained we briefly describe this algorithm in this section and refer the interested reader to [35] for further implementation details.

\subsection{Optimality conditions in the IPM}

The OPF formulations of the previous section can be compactly written as a general nonlinear programming problem:

$\min f(\mathbf{x})$,

s.t. $\mathbf{g}(\mathbf{x})=\mathbf{0}$,

$\mathbf{h}(\mathbf{x}) \geq \mathbf{0}$, where $f(\mathbf{x}), \mathbf{g}(\mathbf{x})$ and $\mathbf{h}(\mathbf{x})$ are assumed to be twice continuously differentiable, $\mathbf{x}$ is a $(\operatorname{dim}(C)+2 n)$-dimensional vector that encompasses both control variables (vector of size $\operatorname{dim}(C)$ ) and state variables (real and imaginary part of voltage at all buses), $\mathbf{g}$ is a $p$-dimensional vector of functions and $\mathbf{h}$ is a $q$-dimensional vector of functions.

The Lagrangian associated with this NLP problem within the IP framework can be defined as:

$L_{\mu}(\mathbf{y})=f(\mathbf{x})-\mu \sum_{i=1}^{q} \ln s_{i}-\boldsymbol{\lambda}^{T} \mathbf{g}(\mathbf{x})-\boldsymbol{\pi}^{T}[\mathbf{h}(\mathbf{x})-\mathbf{s}]$,

where $\mathbf{s}=\left[s_{1}, \ldots, s_{q}\right]^{T}$ is the vector of slack variables, $\boldsymbol{\lambda}$ and $\boldsymbol{\pi}$ are the vectors of Lagrange multipliers (also called dual variables), $\mu$ is a positive scalar called barrier parameter, and $\mathbf{y}=[\mathbf{s} \boldsymbol{\pi} \boldsymbol{\lambda} \mathbf{x}]^{T}$ groups all variables.

The perturbed KKT first order necessary optimality conditions are obtained by setting to zero the derivatives of the Lagrangian with respect to all unknowns [8]:

$\left[\begin{array}{c}\nabla_{\mathbf{s}} L_{\mu}(\mathbf{y}) \\ \nabla_{\boldsymbol{\pi}} L_{\mu}(\mathbf{y}) \\ \nabla_{\boldsymbol{\lambda}} L_{\mu}(\mathbf{y}) \\ \nabla_{\mathbf{x}} L_{\mu}(\mathbf{y})\end{array}\right]=\left[\begin{array}{c}-\mu \mathbf{e}+\mathbf{S} \boldsymbol{\pi} \\ -\mathbf{h}(\mathbf{x})+\mathbf{s} \\ -\mathbf{g}(\mathbf{x}) \\ \nabla f(\mathbf{x})-\mathrm{J}_{\mathrm{g}}(\mathbf{x})^{T} \boldsymbol{\lambda}-\mathrm{J}_{\mathrm{h}}(\mathbf{x})^{T} \boldsymbol{\pi}\end{array}\right]=\mathbf{0}$,

where $\mathbf{S}$ is a diagonal matrix of slack variables, $\mathbf{e}=[1, \ldots, 1]^{T}, \nabla f(\mathbf{x})$ is the gradient of $f, \mathbf{J}_{g}(\mathbf{x})$ is the Jacobian of $\mathbf{g}(\mathbf{x})$ and $\mathbf{J}_{h}(\mathbf{x})$ is the Jacobian of $\mathbf{h}(\mathbf{x})$. Note that in order to facilitate the presentation we have rewritten the complementarity slackness constraints as $\mu \mathbf{e}+\mathbf{S} \pi=\mathbf{0}$ but a proper implementation should rely on the constraints $\mu / \mathbf{s}+\pi=\mathbf{0}$ so as to preserve the symmetry of the Hessian.

The pure primal-dual IP algorithm consists in solving iteratively the linearized KKT conditions for the Newton direction $\Delta \mathbf{y}^{k}$ while decreasing the barrier parameter $\mu^{k}$ gradually to zero as iterations progress:

$\mathbf{H}\left(\mathbf{y}^{k}\right)\left[\begin{array}{c}\Delta \mathbf{s}^{k} \\ \Delta \boldsymbol{\pi}^{k} \\ \Delta \boldsymbol{\lambda}^{k} \\ \Delta \mathbf{x}^{k}\end{array}\right]=\left[\begin{array}{c}\mu^{k} \mathbf{e}-\mathbf{S}^{k} \pi^{k} \\ \mathbf{h}\left(\mathbf{x}^{k}\right)-\mathbf{s}^{k} \\ \mathrm{~g}\left(\mathrm{x}^{k}\right) \\ -\nabla f\left(\mathbf{x}^{k}\right)+\mathrm{J}_{\mathrm{g}}\left(\mathbf{x}^{k}\right)^{T} \boldsymbol{\lambda}^{k}+\mathrm{J}_{\mathrm{h}}\left(\mathbf{x}^{k}\right)^{T} \boldsymbol{\pi}^{k}\end{array}\right]$

where $\mathbf{H}\left(\mathbf{y}^{k}\right)$ is the Hessian matrix (of second derivatives) $\left(\partial^{2} L_{\mu}\left(\mathbf{y}^{k}\right) / \partial \mathbf{y}^{2}\right)$. We denote by $\operatorname{dim}(K K T)$ the size of this Hessian.

\subsection{The MCC algorithm}

We briefly outline the MCC algorithm to solve the KKT optimality conditions (17):

\section{Initialization.}

Set the iteration count $k=0$. Chose $\mu^{0}>0$. Initialize $\mathbf{y}^{0}$, taking care that slack variables and their corresponding dual variables are strictly positive $\left(\mathbf{s}^{0}, \boldsymbol{\pi}^{0}\right)>\mathbf{0}$.

\section{The predictor step.}

(a) Solve the system (18) for the affine-scaling direction, obtained by neglecting $\mu$ in its right-hand side:

$$
\mathbf{H}\left(\mathbf{y}^{k}\right)\left[\begin{array}{c}
\Delta \mathbf{s}_{a f}^{k} \\
\Delta \boldsymbol{\pi}_{a f}^{k} \\
\Delta \boldsymbol{\lambda}_{a f}^{k} \\
\Delta \mathbf{x}_{a f}^{k}
\end{array}\right]=\left[\begin{array}{c}
-\mathbf{S}^{k} \pi^{k} \\
\mathbf{h}\left(\mathbf{x}^{k}\right)-\mathbf{s}^{k} \\
\mathbf{g}\left(\mathbf{x}^{k}\right) \\
-\nabla f\left(\mathbf{x}^{k}\right)+\mathrm{J}_{\mathrm{g}}\left(\mathbf{x}^{k}\right)^{T} \boldsymbol{\lambda}^{k}+\mathrm{J}_{\mathrm{h}}\left(\mathbf{x}^{k}\right)^{T} \boldsymbol{\pi}^{k}
\end{array}\right]
$$


(b) Compute the affine complementarity gap $\rho_{a f}^{k}$ : $\rho_{a f}^{k}=\left(\mathbf{s}^{k}+\alpha_{a f}^{k} \Delta \mathbf{s}_{a f}^{k}\right)^{T}\left(\boldsymbol{\pi}^{k}+\alpha_{a f}^{k} \Delta \boldsymbol{\pi}_{a f}^{k}\right)$ where $\alpha_{a f}^{k} \in(0,1]$ is the step length which would be taken along the affine scaling direction if the latter was used (19).

(c) Estimate the barrier parameter for the next iteration: $\mu_{a f}^{k}=\min \left\{\left(\frac{\rho_{a f}^{k}}{\rho^{k}}\right)^{2}, 0.2\right\} \frac{\rho_{a f}^{k}}{q}$

where $\rho^{k}=\left(\mathbf{s}^{k}\right)^{T} \boldsymbol{\pi}^{k}$ denotes the complementarity gap at the current iterate.

\section{The corrector step.}

(a) Compute a trial ${ }^{2}$ point $\tilde{\mathbf{y}}^{k}=\mathbf{y}^{k}+\tilde{\alpha}^{k} \Delta \mathbf{y}_{a f}^{k}$, where $\tilde{\alpha}^{k}=$ $\min \left(\alpha_{a f}^{k}+\delta_{\alpha}, 1\right)$ with the desired improvement on the step length $\delta_{\alpha}=0.2$.

(b) Compute the complementarity products at the trial point $\tilde{\mathbf{v}}^{k}=\tilde{\mathbf{S}}^{k} \tilde{\pi}^{k}$

(c) Identify components of $\tilde{\mathbf{v}}^{k}$ that do not belong to the interval $\left[\beta_{\min } \mu_{a f}^{k}, \beta_{\max } \mu_{a f}^{k}\right]$, called outlier complementarity products, where $\beta_{\min }=0.1$ and $\beta_{\max }=10$.

(d) Because the corrector step effort focuses on correcting the outliers only in order to improve the centrality of the next iterate, some target complementary products $\left(\tilde{\mathbf{v}}^{k}\right)^{t}$ are defined:

$$
\left(v_{i}^{k}\right)^{t}= \begin{cases}\beta_{\min } \mu_{a f}^{k}, & \text { if } \tilde{v}_{i}^{k}<\beta_{\min } \mu_{a f}^{k} \\ \beta_{\max } \mu_{a f}^{k}, & \text { if } \tilde{v}_{i}^{k}>\beta_{\max } \mu_{a f}^{k} \\ \tilde{v}_{i}^{k}, & \text { otherwise }\end{cases}
$$

(e) The corrector direction $\Delta \mathbf{y}_{c o}^{k}$ is obtained as the solution of the following linear system:

$$
\mathbf{H}\left(\mathbf{y}^{k}\right)\left[\begin{array}{c}
\Delta \mathbf{s}_{c o}^{k} \\
\Delta \boldsymbol{\pi}_{c o}^{k} \\
\Delta \boldsymbol{\lambda}_{c o}^{k} \\
\Delta \mathbf{x}_{c o}^{k}
\end{array}\right]=\left[\begin{array}{c}
\left(\mathbf{v}^{k}\right)^{t}-\tilde{\mathbf{v}}^{k} \\
\mathbf{0} \\
\mathbf{0} \\
\mathbf{0}
\end{array}\right]
$$

where the nonzero components of the right-hand-side correspond to the outlier complementarity products only.

(f) Update ${ }^{3}$ the new search direction:

$$
\Delta \mathbf{y}^{k}=\Delta \mathbf{y}_{a f}^{k}+\Delta \mathbf{y}_{c o}^{k}
$$

(g) Choice of the step length.

Determine the maximum step length $\alpha_{p}^{k} \in(0,1]$ (respectively $\left.\alpha_{d}^{k} \in(0,1]\right)$ in the primal (respectively dual) variables space along the Newton direction $\Delta \mathbf{y}^{k}$ such that $\mathbf{s}^{k+1}>\mathbf{0}$ (respectively $\boldsymbol{\pi}^{k+1}>\mathbf{0}$ ):

$\alpha_{p}^{k}=\min \left\{1, \gamma \min _{\Delta s_{i}^{k}<0} \frac{-s_{i}^{k}}{\Delta s_{i}^{k}}\right\} \quad \alpha_{d}^{k}=\min \left\{1, \gamma \min _{\Delta \pi_{i}^{k}<0} \frac{-\pi_{i}^{k}}{\Delta \pi_{i}^{k}}\right\}$

where $\gamma \in(0,1)$ is a safety factor aiming to ensure strict positiveness of slack variables and their corresponding dual variables. A typical value of the safety factor is $\gamma=0.99995$.

(h) Update solution:

$$
\begin{array}{ll}
\mathbf{s}^{k+1}=\mathbf{s}^{k}+\alpha_{p}^{k} \Delta \mathbf{s}^{k} & \boldsymbol{\pi}^{k+1}=\boldsymbol{\pi}^{k}+\alpha_{d}^{k} \Delta \boldsymbol{\pi}^{k} \\
\mathbf{x}^{k+1}=\mathbf{x}^{k}+\alpha_{p}^{k} \Delta \mathbf{x}^{k} & \boldsymbol{\lambda}^{k+1}=\boldsymbol{\lambda}^{k}+\alpha_{d}^{k} \Delta \boldsymbol{\lambda}^{k}
\end{array}
$$

\footnotetext{
2 Note that at this point some slack variables and/or their corresponding dual variables may violate the strict positivity conditions $\left(\mathbf{s}^{k}, \boldsymbol{\pi}^{k}\right)>\mathbf{0}$.

3 The corrector step can be applied several times. In such a case, the current
} direction $\Delta \mathbf{y}^{k}$ becomes the predictor for a new corrector.
Table 1

Test system summary.

\begin{tabular}{lllllllll}
\hline$n$ & $g$ & $c$ & $b$ & $l$ & $t$ & $o$ & $a$ & $s$ \\
\hline 8387 & 1865 & 4669 & 14,561 & 12,474 & 2087 & 589 & 84 & 178 \\
\hline
\end{tabular}

(i) Repeat the corrector step a pre-defined number of times as long as the improvement in the step length is satisfactory but inferior to 1 .

(IV) Check convergence.

A (locally) optimal solution is found and the optimization process terminates when: primal feasibility, scaled dual feasibility, scaled complementarity gap and objective function variation from an iteration to the next fall below some tolerances $[9,10,12]$ :

$$
\begin{aligned}
& \left.\max _{i} \max _{i}\left\{-h_{i}\left(\mathbf{x}^{k}\right)\right\},\left\|\mathbf{g}\left(\mathbf{x}^{k}\right)\right\|_{\infty}\right\} \leq \epsilon_{1} \\
& \frac{\left\|\nabla f\left(\mathbf{x}^{k}\right)-\mathrm{J}_{\mathrm{g}}\left(\mathbf{x}^{k}\right)^{T} \boldsymbol{\lambda}-\mathrm{J}_{\mathrm{h}}\left(\mathbf{x}^{k}\right)^{T} \boldsymbol{\pi}^{k}\right\|_{\infty}}{1+\left\|\mathbf{x}^{k}\right\|_{2}+\left\|\boldsymbol{\lambda}^{k}\right\|_{2}+\left\|\boldsymbol{\pi}^{k}\right\|_{2}} \leq \epsilon_{1} \\
& \frac{\rho^{k}}{1+\left\|\mathbf{x}^{k}\right\|_{2}} \leq \epsilon_{2} \\
& \frac{\left|f\left(\mathbf{x}^{k}\right)-f\left(\mathbf{x}^{k-1}\right)\right|}{1+\left|f\left(\mathbf{x}^{k}\right)\right|} \leq \epsilon_{2}
\end{aligned}
$$

where usually $\epsilon_{1}=10^{-4}$ and $\epsilon_{2}=10^{-6}$.

If convergence was not achieved set $k \leftarrow k+1$ and go to step II.

\section{Numerical results}

\subsection{Description of the power system model}

We use a 8387-bus modified model ${ }^{4}$ of the interconnected EHV European power system which spans from Portugal and Spain to Ukraine, Russia and Greece. Notice that in this model the real parameters of the individual power system components (e.g. lines, transformers, etc.), the network topology, as well as the limits on generators active/reactive powers, transformers ratio and angle, voltages, and branch currents have been biased. Nevertheless, this model is representative for the European interconnection in terms of system size and complexity.

In order to assess the robustness of our tool we have chosen very tight operational limits and physical bounds over controls. As a consequence the base case is quite constrained, e.g. many generators have narrow physical active/reactive power limits, many voltage limits are very tight, the angle range of several phase shifters is very small, 6 lines are loaded at more than $90 \%$, etc. These data contain many very short lines (e.g. 98 lines have their reactance lower than $0.0002 \mathrm{pu}$ ) and many artificial lines, stemming from some network equivalents, with very large impedance values, which lead to relatively ill-conditioned problems. For instance for this data set the ratio between the maximum impedance and minimum impedance over all branches is around 408,854, while for the IEEE 118 system [15] (respectively the Poland 2746-bus model [14]) this ratio is around 51 (respectively 15,516). In addition, this data set contains 743 branches with a larger ratio than the maximum ratio of the Poland 2746-bus model.

A summary of the characteristics of this test systems is given in Table 1.

\footnotetext{
${ }^{4}$ More comprehensive data sets of this system are available upon request from the project PEGASE web-page: www.fp7-pegase.eu.
} 
Table 2

Problems definition.

\begin{tabular}{|c|c|c|c|c|c|c|c|c|c|c|c|c|c|c|c|c|}
\hline \multirow[t]{2}{*}{ Problem } & \multirow[t]{2}{*}{ Base case } & \multirow[t]{2}{*}{ Obj. } & \multicolumn{6}{|c|}{ Control variables } & \multicolumn{8}{|c|}{ Inequality constraints } \\
\hline & & & $P_{g}$ & $P_{g}^{s}$ & $V_{g}$ & $r_{1}$ & $B_{S}$ & $\bar{a}$ & $P_{g}$ & $P_{g}^{s}$ & $Q_{g}$ & $r_{1}$ & $B_{S}$ & $a$ & $V$ & I \\
\hline P1 & A & MPL & & $x$ & & & & & & $x$ & $x$ & & & & $x$ & \\
\hline P2 & A & MPL & & $\times$ & $\times$ & $\times$ & $x$ & & & $\times$ & $x$ & $x$ & $\times$ & & $\times$ & \\
\hline P3 & $\mathrm{A}$ & MPL & & $\times$ & $\times$ & $\times$ & $x$ & & & $\times$ & $\times$ & $\times$ & $x$ & & $\times$ & $x$ \\
\hline P4 & A & MD & $x$ & & & & & & $x$ & & $x$ & & & & & $x$ \\
\hline P5 & A & MD & $x$ & & & & & & $x$ & & $x$ & & & & $x$ & $x$ \\
\hline P6 & A & MD & $x$ & & & $\times$ & $x$ & & $x$ & & $\times$ & $x$ & $x$ & & $\times$ & $x$ \\
\hline P7 & A & MD & $x$ & & & $x$ & $x$ & $\times$ & $x$ & & $x$ & $x$ & $x$ & $x$ & $\times$ & $\times$ \\
\hline P8 & A & MD & $x$ & & $\times$ & $\times$ & $x$ & & $x$ & & $\times$ & $\times$ & $x$ & & $\times$ & $\times$ \\
\hline P9 & A & $\mathrm{MD}$ & $x$ & & $x$ & $x$ & $x$ & $\times$ & $x$ & & $x$ & $x$ & $x$ & $x$ & $x$ & $x$ \\
\hline P10 & B & $\mathrm{MD}$ & $x$ & & $x$ & & & & $x$ & & $\times$ & & & & $\times$ & $x$ \\
\hline P11 & B & MD & $x$ & & $x$ & $x$ & $x$ & & $x$ & & $x$ & $x$ & $x$ & & $\times$ & $\times$ \\
\hline P12 & B & MD & $x$ & & $x$ & $x$ & $x$ & $x$ & $x$ & & $x$ & $x$ & $x$ & $x$ & $x$ & $x$ \\
\hline
\end{tabular}

\subsection{Simulation assumptions}

We consider in our simulations two base cases denoted as A and $B$. In the base case A some voltage limits are not met. The base case $B$ is the same as A except that the flow limit of a line has been decreased to create a thermal congestion.

Unless otherwise specified, we use the default settings of the MCC algorithm [35] in all computations to enable a fair assessment of the performances of the algorithm.

All tests have been performed on a PC of 2.8-GHz and 4-GB RAM, using the IPM-based NLP OPF program, developed for research purposes by the authors in C++within the Cygwin environment [35].

\subsection{OPF problems definition}

In order to test the robustness and efficiency of the IPM, several OPF problems have been solved involving different combinations of objectives, controls and constraints, as shown in Table 2. In this table $P_{g}, P_{g}^{s}, V_{g}, Q_{g}, r_{1}, B_{s}, a, I$, and $V$ refer to generator active power, slack generator active power, generator terminal voltage, generator reactive power, controllable transformer ratio, shunt susceptance, phase shifter angle, branch current, and bus voltage magnitude, respectively, while symbol " $\times$ " means that the variable/constraint is considered in the OPF.

In all problems the equality constraints are the power-flow equations and sometimes phase shifter angles (e.g. in P7, P9, and P12) and generators voltages (e.g. in P4 to P7).

\subsection{Observations about the OPF results}

Table 3 provides for each problem the size $\operatorname{dim}(C)$ of the set of control variables, the size $\operatorname{dim}(K K T)$ of the full system of KKT optimality constraints (17), and the number of iterations to

Table 3

Problems size and convergence details.

\begin{tabular}{llrrr}
\hline Problem & $\operatorname{dim}(C)$ & $\operatorname{dim}(K K T)$ & Iter. & Time $(\mathrm{s})$ \\
\hline P1 & 1825 & 76,221 & 43 & 16.0 \\
P2 & 2592 & 80,056 & 45 & 16.8 \\
P3 & 2592 & 105,004 & 65 & 47.4 \\
P4 & 1250 & 72,204 & 9 & 5.0 \\
P5 & 1250 & 105,752 & 10 & 8.1 \\
P6 & 2017 & 109,587 & 14 & 10.7 \\
P7 & 2185 & 110,175 & 13 & 10.0 \\
P8 & 4413 & 114,109 & 9 & 8.3 \\
P9 & 4581 & 114,697 & 9 & 7.2 \\
P10 & 3646 & 110,274 & 17 & 12.1 \\
P11 & 4413 & 114,109 & 14 & 10.6 \\
P12 & 4581 & 114,697 & 16 & 50.8 \\
\hline
\end{tabular}

Table 4

Number and type of active constraints for problem P3.

\begin{tabular}{llllll}
\hline \multicolumn{2}{l}{ Active constraints } & & & & Total \\
\hline$Q_{g}$ & $I$ & $V$ & $r_{1}$ & $B_{s}$ & \\
\hline 330 & 18 & 1064 & 83 & 63 & 1558 \\
\hline
\end{tabular}

convergence as well as the corresponding CPU times ${ }^{5}$ (in seconds). Furthermore, in general the larger the number of active constraints the larger the number of iterations to convergence and hence the computational time. Notice that the convergence is achieved within a reasonable CPU time for all problems in spite of the large size of the system and all the computational challenges mentioned previously. The average computational effort per iteration generally increases with the problem size while the overall computing time seems to be rather more influenced by the problem size than by the number of control variables.

Table 4 shows the number and the type of active constraints at the optimum of problem P3.

The very large number of 1558 active constraints confirms the extreme difficulty of the problem. The algorithm's ability to effectively coordinate 18 active line current constraints (and 3 other lines loaded above $96 \%$ ) together with $1064 \mathrm{~V}$ limits is remarkable. Notice that most NLP OPF test scenarios of the literature, and in particular for IPM-based OPFs, rarely have more than a few active line-current constraints and a few hundred of other active constraints (see however [30], a work on SLP-based SCOPF, where 23 line current constraints are active).

\subsection{OPF failure on a very tough problem}

We now focus on problem P8 but minimize the overall active power generation and hence replace the quadratic objective (1) by the linear one:

$L=\min \sum_{i=1}^{g} P_{g i}$.

For this problem the MCC algorithm gets stuck on an infeasible non-optimal point. Furthermore, the convergence is not restored even after trying few classical heuristic techniques (e.g. different initial values for the barrier parameter $\mu$, smaller values of the safety parameter $\gamma$, and different initializations of variables) $[16,40]$. However, by using another IPM algorithm, namely the predictor-corrector (that we generally found slightly less reliable than the MCC algorithm) a locally optimal solution is found. Table 5

\footnotetext{
${ }^{5}$ CPU time concerns the optimization process only.
} 
Table 5

Number and type of active constraints for the modified problem P8.

\begin{tabular}{lllllll}
\hline \multicolumn{2}{l}{ Active constraints } & \multicolumn{1}{c}{} & & Total \\
\hline$P_{g}$ & $Q_{g}$ & $I$ & $V$ & $r_{1}$ & $B_{s}$ & \\
\hline 828 & 379 & 76 & 510 & 58 & 80 & 1931 \\
\hline
\end{tabular}

Table 6

SCOPF statistics for problem P2.

\begin{tabular}{llrlc}
\hline Number contingencies & $\operatorname{dim}(C)$ & $\operatorname{dim}(K K T)$ & Iter. & Time $(\mathrm{s})$ \\
\hline 0 & 2592 & 80,056 & 45 & 16.8 \\
1 & 4417 & 158,101 & 53 & 78.5 \\
2 & 6242 & 236,146 & 55 & 155.0 \\
3 & 8067 & 314,191 & 75 & 389.0 \\
\hline
\end{tabular}

Table 7

SCOPF statistics for problem P8.

\begin{tabular}{lrccc}
\hline Number contingencies & $\operatorname{dim}(C)$ & $\operatorname{dim}($ KKT $)$ & Iter. & Time $(\mathrm{s})$ \\
\hline 0 & 4413 & 114,109 & 9 & 8.3 \\
1 & 6238 & 217,100 & 10 & 30.2 \\
2 & 8063 & 320,091 & 15 & 93.0 \\
3 & 9888 & 423,082 & 11 & 140.9 \\
4 & 11,713 & 526,673 & 10 & 204.3 \\
\hline
\end{tabular}

provides the number and the type of active constraints at this optimum. The choice of this linear objective leads, as expected, to a high number of active power generator constraints active at the optimum. The very large total number of 1931 active constraints, as well as the large number of 76 active branch current constraints (plus 11 lines loaded above 95\% at the optimum), indicate again the toughness of this test scenario as compared with those from the literature.

\subsection{SCOPF solutions}

In order to further assess how the IPM scales with problem size we consider the problems P2 and P8 solved by a SCOPF in preventive only mode [2]. The latter consists in duplicating base case constraints (3)-(13) for each contingency included in the SCOPF and assumes that control variables have the same value in both base case and contingency states. This SCOPF is augmented with one contingency at the time.

Tables 6 and 7 provide for each problem the size of the set of control variables, the size of the full system of KKT optimality constraints, the number of iterations to convergence and the CPU times. One can observe that for both problems the computational effort increases much more than linearly with the size but still remains reasonable given the problem size. Furthermore, as in previous examples, we observe that the size of the problem has a more significant impact on the computational effort than the number of control variables.

\subsection{Comparison with available solvers}

In this section we perform a comparison with the two most efficient alternative available solvers for large scale systems on MatPower Version 4.1 running under Matlab 7.13 [22], namely the Matlab Interior Point Solver (MIPS) and the Primal Dual Interior Point Method (PDIPM). Although MATPOWER possesses several interesting less conventional model features, the current version does not allow modelling any among our twelve more classical OPF problems in terms of objective function and control variables. In order to enable an as fair as possible comparison, we consider only the OPF objective function of minimum generation cost/deviation, generators active/reactive powers as control variables, and
Table 8

OPF comparison with MATPOWER.

\begin{tabular}{lllll}
\hline Base case & Obj. & $\begin{array}{l}\text { Our solver } \\
\text { it/time }\end{array}$ & $\begin{array}{l}\text { MIPS } \\
\text { it/time }\end{array}$ & $\begin{array}{l}\text { PDIPM } \\
\text { it/time }\end{array}$ \\
\hline A & L & $97 / 71.9$ & $66 / 31.8$ & $76 / 37.8$ \\
A-PST & L & $346 / 252$ & Failed & Failed \\
A & Q & $13 / 10.7$ & $47 / 22.1$ & Failed \\
A-PST & Q & $20 / 15.7$ & $32 / 17.8$ & $42 / 20.6$ \\
\hline
\end{tabular}

constraints on branch currents, voltage magnitude and physical limits on generators' active/reactive powers. Still there remain two slight modelling differences concerning the transformer transversal susceptance and the handling of current constraints, as our solver implements only constraints on longitudinal branch currents (7), whereas MATPOWER solvers implement such constraints at both ends of each branch. Furthermore, PDIPM implements only MVA flow constraints. However, as the main computational burden in IPM is the factorization of a linear system of equations [12], we notice that as MATPOWER solvers rely on the "reduced KKT system" $[10,21]$, the number of inequality constraints does not affect the size of this system, contrary to our implementation which uses the full KKT system (17).

Table 8 presents the results of our experiments for four feasible OPF problems, where "L" denotes the linear objective ${ }^{6}$ function (24), "Q" denotes the quadratic objective function MD (1), and the base case A-PST has been obtained from the base case A by setting to zero the angle of all 84 phase shifters and using as starting point the load flow solution of case A.

The results show that both MIPS and PDIPM solvers have a lower computational effort per iteration than our solver, which is certainly due to the use of the reduced KKT system and possibly the library used to solve this system. However, our solver converges in reasonable time and is both faster and needs a lower number of iterations for the quadratic objective. The results also indicate that, similarly to the failure of our solver for the problem described in Section 4.5, other excellent solvers may also occasionally fail, especially on very tough optimization problems as in case A-PST. In the latter case the very large number of iterations of our solver to convergence is not satisfactory and could be practically considered as a case of failure, e.g. if a maximum running time was required. We presume that by running again the solver with different sets of parameters [16] the convergence of failed cases could be restored. A comprehensive comparison concerning the relative reliability of these solvers is out of the scope of the paper, as it should consider many different OPF problems under varied operating conditions. We only underline the need to improve the reliability of IPM codes for very tough large scale OPF problems.

Taking into account the slight modeling differences and the large number of 84 branch current binding constraints, we assessed that in all cases MIPS and our solver converged practically to the same solution.

\subsection{SCOPF solutions on a 3012-bus system}

We consider a model of the Poland power system which OPF data are available on MATPOWER web-site [22]. The system comprises 3012 buses, 3371 lines, 201 transformers, and 298 generators (obtained after aggregating coexisting generators at the same bus).

We consider the problem of minimizing generation cost solved by a SCOPF in corrective mode [37]. We use 292 generators active

\footnotetext{
${ }^{6}$ Notice that for this objective function our solver failed for a more complex OPF problem in terms of controls and constraints (see Section 4.5).
} 
Table 9

SCOPF results for the Poland system.

\begin{tabular}{ccrcc}
\hline Number contingencies & $\operatorname{dim}(C)$ & $\operatorname{dim}(K K T)$ & Iter. & Time $(\mathrm{s})$ \\
\hline 0 & 480 & 33,640 & 35 & 6.7 \\
1 & 960 & 68,446 & 38 & 24.4 \\
2 & 1440 & 103,252 & 37 & 60.1 \\
3 & 1920 & 138,058 & 42 & 122.3 \\
4 & 2400 & 172,864 & 41 & 177.2 \\
5 & 2880 & 207,670 & 41 & 68.7 \\
6 & 3360 & 242,476 & 39 & 76.0 \\
7 & 3840 & 277,282 & 40 & 88.2 \\
8 & 4320 & 312,088 & 46 & 113.8 \\
9 & 4800 & 346,894 & 47 & 129.0 \\
10 & 5280 & 381,700 & 47 & 144.9 \\
11 & 5760 & 416,506 & 47 & 158.1 \\
12 & 6540 & 451,312 & 48 & 176.3 \\
\hline
\end{tabular}

power and 188 generators reactive power as control variables, and constraints on branch currents, voltages, physical limits on generators' active/reactive powers, and active power re-dispatch of 292 generators as corrective actions.

Table 9 shows the results obtained with our solver for increasing numbers of contingencies included in the SCOPF. Except maybe for the cases where the number of contingencies included in the SCOPF ranges from 1 to 4 , the algorithm scales very well.

These results show that the SCOPF solution for this already quite large system is reasonably fast and can be envisaged even close to real-time. Furthermore, comparing SCOPF results of Tables 7 and 9 for problems of close sizes one can observe that, the average computational effort per iteration for the 3012-bus system is considerably faster than for the 8387-bus system, which we explain by the much worse numerical conditioning of the latter system, as already discussed in Section 4.1 .

\section{Discussion, conclusions and perspectives}

This paper has presented extensive numerical results with IPM for large scale OPF/SCOPF problems, with the goal of advancing the state of the knowledge about this method in terms of robustness and scalability.

Our results show that nowadays, even without using the most powerful computers available on the market, it is certainly feasible to run an NLP OPF for a large system in real-time provided that a reliable solver is used. Furthermore, we prove that our SCOPF code is practical on a 3012-bus system. On the other hand, for the poorly conditioned 8387-bus grid, computer memory limitations prevented us from including more than few contingencies (using the full network model) into the SCOPF, and therefore to assess how the computational effort further scales with the problem size. However, even assuming sufficient computer memory, as one could foresee that for such a system few tens of contingencies might be binding at the optimum, our solver could not comply with close to real-time requirements, while it could possibly still be used in day-ahead operational planning. For such large-scale systems, in order to render the SCOPF tractable in realtime applications one will have to adopt approximate models for post-contingency states $[27,34]$ or resort to Benders decomposition [37].

As the factorization of the Hessian matrix is by far the most expensive computational task of an interior-point algorithm iteration the choice of a suitable library for the solution of a symmetric, very sparse, and very ill-conditioned system of linear equations is paramount. In our implementation we used the sparse objectoriented library SPOOLES [38] which has several options, and we could experiment the strong dependence of performance vs. robustness tradeoffs on the particular options chosen.
Furthermore, in our implementation, for the sake of facilitating the programming effort, we solve at each iteration the full system of KKT optimality conditions whereas most authors recommend the use of the "reduced system" only [10], which may break-down the size of the system up to around 30\% by appropriate elimination of the complementarity slackness and inequality constraints. Although the complementarity slackness constraints are trivial equations and therefore should not pose additional problems to a smart library, a further decrease of the computational time should be expected if one relies on the "reduced system" only.

Our study also shows that the IPM algorithms proposed in the power systems literature may lead to convergence problems on very hard optimization problems. The main cause of convergence problems of the IPM is that the iterations becomes stuck at a non-optimal point if one approaches too early the feasibility boundary and therefore the slack variables prematurely go to 0 , a phenomenon called jamming [39]. Fortunately, there are some well-known remedies to restore the convergence of an IPM (e.g. changing the initial value of some parameters and especially the barrier parameter, using a less aggressive decrease of the barrier parameter, using an alternative interior-point algorithm, etc.) $[16,40]$, but they take additional computational effort without guaranteeing solution restoration.

We envisage to improve the reliability of our IPM implementation by focusing on three aspects: the use of anti-jamming remedies [39], regularization (or to ensure the smoothness of the solution by augmenting the problem with penalty functions to better deal with an ill-posed Hessian matrix) [41], and use of merit functions to ensure that joint progress is made both towards a local minimizer and towards feasibility (this progress is achieved by shortening the step-length along the Newton search direction) [42].

A sensible solution to mitigate reliability issues, beyond using appropriate libraries for the solution of linear systems of equations, would be to embed a robust and fast generic NLP solver in the OPF [34]. Nowadays there exist indeed several mature generic NLP solvers (e.g. KNITRO, IPOPT, LOQO, CONOPT, SNOPT, MINOS, etc.) that proved excellent performances on generic problems as well as for some power system problems modeled in AMPL [43] or GAMS [44].

Using OPF/SCOPF in real-time involves among others the successive solutions of closely related NLP problems. The IPM does not naturally warm start well and hence cannot directly take advantage of the solutions gotten at the previous time-steps. Although the current speed of IPM is satisfactory since, as with any Newtonbased method the number of iterations generally scales acceptably with the problem size, any improvement of its warm start ability (or, more generally, of its learning ability) is welcome. We look forward to assess on SCOPF problems the improvements reported for generic NLPs (see e.g. [41]). Furthermore, recent results [45] show that methods supposed to warm start well (e.g. SQP) may fail on large scale problems with a very large number of binding constraints at the optimum: in particular, it was found that for NLP OPFs with equilibrium constraints (in which equilibrium constraints are modeled by RNS functions) the warm start of KNITRO (with SQP option) failed while the SQP solver SNOPT did not perform faster than the cold start of KNITRO (with IPM option); also, the SQP solver SNOPT failed on cold start on most problems, while KNITRO (with IPM option) was found the most reliable among the solvers tested [45].

Finally, the present paper focused on NLP aspects of the OPF problem, but the impact on the overall reliability and speed of the OPF process of other key features (e.g. handling of discrete variables, use of limited numbers of control actions, etc.) remain also to be carefully assessed $[4,5,46,47]$. In addition, improved ways to analyze and visualize the results of large-scale OPF, e.g. in case of grid congestion, should be devised [30]. 


\section{Acknowledgments}

The authors would like to thank RTE France (the French transmission system operator) for their support and advices on the problem formulation.

Florin Capitanescu is supported by the National Research Fund Luxembourg in the frame of the CORE project "Reliable and efficient distributed electricity generation in smart grids", C11/SR/1278568.

Louis Wehenkel acknowledges the support of the Belgian Network DYSCO, funded by the Interuniversity Attraction Poles Programme, initiated by the Belgian State, Science Policy Office. The scientific responsibility rests with the authors.

We thank Cleve Ashcraft, the main developer of the SPOOLES library, for useful suggestions concerning the proper use of the library for optimization problems.

We thank Ray Zimmerman, the main developer of MATPOWER, for useful suggestions concerning the comparisons made with MATPOWER.

\section{References}

[1] J. Carpentier, Contribution à l'étude du dispatching économique, Bulletin de la Société Française d'Electricité 3 (1962) 431-447.

[2] O. Alsac, B. Stott, Optimal load flow with steady-state security, IEEE Transactions on Power Apparatus and Systems PAS-93 (3) (1974) 745-751.

[3] M. Ilic, AC OPF and smart grids, in: presentation at FERC Conference on Enhanced Optimal Power Flow Models, Washington, USA, 2010.

[4] B. Stott, O. Alsac, A.J. Monticelli, Security analysis and optimization, Proceedings of the IEEE 75 (12) (1987) 1623-1644 (Invited paper).

[5] A. Papalexopoulos, Challenges to on-line OPF implementation, in: IEEE/PES Winter Meeting, New York, USA, 1995.

[6] CORESO (Regional Coordination Service Centre), http://www.coreso.eu

[7] K.R. Frisch, The logarithmic potential method of convex programming, Manuscript at Institute of Economics, University of Oslo, Norway, 1955.

[8] A.V. Fiacco, G.P. McCormick, Nonlinear Programming: Sequential Unconstrained Minimization Techniques, John Willey \& Sons, New York, 1968.

[9] Y.C. Wu, A.S. Debs, R.E. Marsten, A direct nonlinear predictor-corrector primal-dual interior point algorithm for optimal power flows, IEEE Transactions on Power Systems 9 (2) (1994) 876-883.

[10] S. Granville, Optimal reactive dispatch through interior point methods, IEEE Transactions on Power Systems 9 (1) (1994) 136-142.

[11] G.D. Irrisari, X. Wang, J. Tong, S. Mokhtari, Maximum loadability of power systems using interior point nonlinear optimization methods, IEEE Transactions on Power Systems 12 (1) (1997) 162-172.

[12] G.L. Torres, V.H. Quintana, An interior-point method for nonlinear optimal power flow using rectangular coordinates, IEEE Transactions on Power Systems 13 (4) (1998) 1211-1218.

[13] H. Wei, H. Sasaki, J. Kubokawa, R. Yokohama, An interior point nonlinear programming for optimal power flow with a novel data structure, IEEE Transactions on Power Systems 13 (3) (1998) 870-877.

[14] Data of the Poland power system, available online at the MATPOWER ("A MATLAB Power System Simulation Package" by R.D. Zimmerman, C.E. Murillo-Sanchez, and Deqiang Gan), web-page: http://www.pserc.cornell.edu/matpower/

[15] Data of IEEE 300-Bus System, 1996, Available from: http://www.ee. washington.edu

[16] G.L. Torres, Nonlinear optimal power flow by interior and non-interior point methods, PhD Thesis, University of Waterloo, Canada, 1998.

[17] G.L. Torres, V.H. Quintana, On a nonlinear multiple centrality corrections interior-point method for optimal power flow, IEEE Transactions on Power Systems 16 (2) (2001) 222-228.

[18] M.J. Rider, C.A. Castro, V.L. Paucar, A.V. Garcia, Higher order interior-point method for minimising load-shedding in a competitive electric power market, IEE Proceedings Generation, Transmission \& Distribution 151 (4) (2004) 433-440.

[19] M.J. Rider, C.A. Castro, M.F. Bedrinana, A.V. Garcia, Towards a fast and robust interior point method for power system applications, IEE Proceedings Generation, Transmission \& Distribution 151 (5) (2004) 575-581.

[20] Q.Y. Jiang, H.-D. Chiang, C.X. Guo, Y.J. Cao, Power-current hybrid rectangular formulation for interior-point optimal power flow, IET Generation Transmission \& Distribution 3 (8) (2009) 748-756.
[21] H. Wang C.E. Murillo-Sanchez, R. Zimmerman, RJ. Thomas, On computational issues of market-based optimal power flow, IEEE Transactions on Power Systems 22 (3) (2007) 1185-1192.

[22] R. Zimmerman, C.E. Murillo-Sanchez, R.J. Thomas, MatPoweR: steady-state operations, planning, and analysis tools for power systems research and education, IEEE Transactions on Power Systems 26 (1) (2011) 12-19.

[23] S. Hao, A. Papalexopoulos, External network modeling for optimal power flow applications, IEEE Transactions on Power Systems 10 (2) (1995) 825-837.

[24] G.L. Torres, V.H. Quintana, Optimal power flow by a nonlinear complementarity method, IEEE Transactions on Power Systems 15 (3) (2000) 1028-1033.

[25] V.A. de Sousa, E.C. Baptista, G.R.M. da Costa, Optimal reactive power flow via the modified barrier Lagrangian function approach, Electric Power Systems Research 84 (1) (2012) 159-164.

[26] V.A. de Sousa, E.C. Baptista, G.R.M. da Costa, Loss minimization by the predictor-corrector modified barrier approach, Electric Power Systems Research 79 (5) (2009) 803-808.

[27] O. Alsac, J. Bright, M. Prais, B. Stott, Further developments in LP-based optimal power flow, IEEE Transactions on Power Systems 5 (3) (1990) 697-711.

[28] T.J. Bertram, K.D. Demaree, L.C. Dangelmaier, An integrated package for realtime security enhancement, IEEE Transactions on Power Systems 5 (2) (1990) $592-600$

[29] K. Karoui, L. Platbrood, H. Crisciu, R.A. Waltz, New large-scale security constrained optimal power flow program using a new interior-point algorithm, in: 5-th International Conference on European Electricity Market, 2008.

[30] T.J. Overbye, Xu Cheng, Yan Sun, A comparison of the AC and DC power flow models for LMP calculations, in: Proc. of the 37th Annual HICSS conference, Hawaii, 2004

[31] R. Jabr, N. Martins, B. Pal, S. Karaki, Contingency constrained VAr planning using penalty successive conic programming, IEEE Transactions on Power Systems 27 (1) (2012) 545-553.

[32] A. Marano Marcolini, F. Capitanescu, J.L. Martinez Ramos, L. Wehenkel, Exploiting the use of DC SCOPF approximation to improve iterative AC SCOPF algorithms, IEEE Transactions on Power Systems (2012).

[33] P.J. Macfie, G.A. Taylor, M.R. Irving, P. Hurlock, H.-B. Wan, Proposed shunt rounding technique for large-scale security constrained loss minimization, IEEE Transactions on Power Systems 25 (3) (2010) 1478-1485.

[34] L. Platbrood, H. Crisciu, F. Capitanescu, L. Wehenkel, Combining iterative algorithms and network compression for solving very large scale SCOPF problems, in: PSCC Conference, Sweden, August, 2011.

[35] F. Capitanescu, M. Glavic, D. Ernst, L. Wehenkel, Interior-point based algorithms for the solution of optimal power flow problems, Electric Power Systems Research 77 (5-6) (2007) 508-517.

[36] J. Gondzio, Multiple centrality corrections in a primal-dual method for linear programming, Computational Optimization and Applications 6 (1996) 137-156.

[37] A.J. Monticelli, M.V.P. Pereira, S. Granville, Security-constrained optimal power flow with post-contingency corrective rescheduling, IEEE Transactions on Power Systems 2 (1) (1987) 175-182.

[38] C. Ashcraft, R. Grimes, SPOOLES: an object-oriented sparse matrix library, in: Proceedings of the 1999 SIAM Conference on Parallel Processing for Scientific Computing, 1999, pp. 22-27.

[39] H.Y. Benson, D.F. Shanno, R.J. Vanderbei, Interior-point methods for nonconvex nonlinear programming: jamming and comparative numerical testing, Mathematical Programming, Series A 99 (1) (2004) 35-48

[40] J. Nocedal, A. Waechter, R. Waltz, Adaptive barrier update strategies for nonlinear interior methods, SIAM Journal on Optimization 19 (4) (2009) 1674-1693.

[41] H.Y. Benson, D.F. Shanno, Interior-point methods for nonconvex nonlinear programming: regularization and warmstarts, Computational Optimization and Applications 40 (2) (2008) 143-189.

[42] H.Y. Benson, D.F. Shanno, R.J. Vanderbei, Interior-point methods for nonconvex nonlinear programming: filter methods and merit functions, Computational Optimization and Applications 23 (2) (2002) 257-272.

[43] A Modeling Language for Mathematical Programming (AMPL), Available from: www.ampl.com

[44] B.A. McCarl, GAMS User Guide, Version 23.6, 2011, Available from: www.gams.com

[45] L. Platbrood, S. Fliscounakis, F. Capitanescu, P. Panciatici, C. Merckx, M. Ortega-Vazquez, Deliverable D3.2: development of prototype software for system steady-state optimization of the European transmission system, PEGASE project, 2011, Available from: http://www.fp7-pegase.eu/

[46] B. Stott, O. Alsac, Optimal power flow - basic requirements for real-life problems and their solutions, in: SEPOPE XII Symposium, Rio de Janeiro, Brazil, May 2012 (White paper)

[47] F. Capitanescu, J.L. Martinez Ramos, P. Panciatici, D. Kirschen, A. Marano Marcolini, L. Platbrood, L. Wehenkel, Security-constrained optimal power flow: state-of-the-art, challenges, and future trends, Electric Power Systems Research 81 (8) (2011) 1731-1741. 